\title{
Sunset integrals at finite volume
}

\section{Johan Bijnens*}

Deaprtment of Astronomy and Theoretical Physics, Lund University, Sölvegatan 14A, SE 22362 Lund, Sweden

E-mail: bijnensethep.lu.se

Chiral Perturbation Theory is a useful tool to aid in performing the various extrapolations needed in lattice QCD calculations of physical quantities. These include extrapolations in quark mass, finite lattice spacing and finite size of the lattice. Especially the latter will become more important when the quark masses on the lattice become smaller.

Here we develop the needed two-loop integrals at finite volume to do the calculations for masses and decay constants for all general mass cases.

I will present results based on an expansion in Bessel functions as well as on a version using theta functions and compare their efficiency. Work is in progress to combine these results with two-loop ChPT calculations.

31st International Symposium on Lattice Field Theory LATTICE 2013

July 29 - August 3, 2013

Mainz, Germany

${ }^{*}$ Speaker. 


\section{Motivation}

Lattice QCD calculates at different quark masses and volumes and Chiral Perturbation Theory has been very useful in the past for doing the extrapolations in quark masses. Masses and decay constants are known to two-loop order for the two- and three-flavour case as well as for all mass cases with partial quenching [1-3]. At finite volume as a pure ChPT calculation the mass for the two-flavour case [5] and the vacuum expectation value in the three flavour case is known [4]. The reason why we want to study also the general mass case is that the Compton wavelength of the pion is about $1.4 \mathrm{fm}$ and one might therefore have to go beyond the leading large volume $e^{-m_{\pi} L}$ terms with with present lattices. The convergence of Chiral Perturbation Theory is governed by the resonance scale of $1 / m_{\rho} \approx 0.25 \mathrm{fm}$.

In the remainder I will work with an infinite extension in the time direction and a size $L$ in the three spatial directions. The first work on finite volume corrections in this context was done in [6]. There is a large volume of work at one-loop order but at two-loop only the above quoted exists.

I will first explain in detail the simplest one-loop case, tadpole integrals and then introduce the extra parts needed to do the two-loop sunset integrals.

This work will be published in [7]. Some partial results ${ }^{1}$ are in the master thesis [8].

\section{Underlying formulas}

In a finite volume the Fourier transform becomes a Fourier sum instead. Let me first illustrate it in one dimension with periodic boundary conditions $F(x+L)=F(x)$ :

$$
\int \frac{d p}{2 \pi} F(p) \longrightarrow \frac{1}{L} \sum_{p_{n}=2 \pi n / L} F\left(p_{n}\right) \equiv \int_{L} \frac{d p}{2 \pi} F(p) .
$$

The integral with the subscript is defined to mean the sum. The problem is that the sum is not simple to regulate when you have divergences. The Poisson summation formula allows to again bring in an integral

$$
\frac{1}{L} \sum_{p_{n}=2 \pi n / L} F\left(p_{n}\right)=\sum_{\ell=n L} \int \frac{d p}{2 \pi} e^{i \ell p} F(p) .
$$

If a twist angle $\theta$ is introduced in the boundary condition $\theta, \phi(x+L)=e^{i \theta} \phi(x)$, we get instead a sum over $p_{n}=(2 \pi / L) n+(\theta / L)$ and

$$
\frac{1}{L} \sum_{p_{n}=2 \pi n / L+\theta / L} F\left(p_{n}\right)=\sum_{\ell=n L} \int \frac{d p}{2 \pi} e^{i(\ell p-\ell(\theta / L))} F(p) .
$$

\section{One-loop tadpole}

Let me now illustrate the procedure on the simplest loop-integral, the one-loop tadpole:

$$
\lfloor X\rfloor=\int_{V} \frac{d^{d} r}{(2 \pi)^{d}} \frac{X}{\left(r^{2}+m^{2}\right)^{n}} .
$$

\footnotetext{
${ }^{1}$ Note that there are misprints in both [8] and my slides at the conference.
} 
We do the Poisson trick in the three spatial dimensions

$$
\lfloor X\rfloor=\sum_{l_{r}} \int \frac{d^{d} r}{(2 \pi)^{d}} \frac{X e^{i l_{r} \cdot r-i l_{r} \cdot \Theta}}{\left(r^{2}+m^{2}\right)^{n}},
$$

with $l_{r}=\left(0, n_{1} L, n_{2} L, n_{3} L\right), \Theta=(0, \vec{\theta} / L)$. We split of the term with $l_{r}=0$, i.e. the infinite volume term, and the rest with $\lfloor X\rfloor=\lfloor X\rfloor^{\infty}+\lfloor X\rfloor^{V}$. The denominator can be brought up with ' $\alpha$ ' parameters, $1 / a=\int_{0}^{\infty} d \lambda e^{-\lambda a}$.

$$
\lfloor 1\rfloor^{V}=\frac{1}{\Gamma(n)} \sum_{l_{r}}^{\prime} \int \frac{d^{d} r}{(2 \pi)^{d}} \int_{0}^{\infty} d \lambda \lambda^{n-1} e^{i l_{r} \cdot r-i l_{r} \cdot \Theta} e^{-\lambda\left(r^{2}+m^{2}\right)}
$$

$\sum_{l_{r}}^{\prime}$ means sum without $l_{r}=0$ (all components zero). We shift the integration momentum by $r=$ $\bar{r}+i l_{r} /(2 \lambda)$ to obtain $\lfloor 1\rfloor^{V}=\frac{1}{\Gamma(n)} \sum_{l_{r}}^{\prime} \int_{0}^{\infty} d \lambda \lambda^{n-1} e^{-\lambda m^{2}-\frac{l_{r}^{2}}{4 \lambda}-i l_{r} \cdot \Theta} \int \frac{d^{d} \bar{r}}{(2 \pi)^{d}} e^{-\lambda \bar{r}^{2}}$ This finally leads to the master formulae for tadpoles:

$$
\lfloor 1\rfloor^{V}=\frac{1}{(4 \pi)^{d / 2} \Gamma(n)} \sum_{l_{r}}^{l} \int_{0}^{\infty} d \lambda \lambda^{n-\frac{d}{2}-1} e^{-\lambda m^{2}-\frac{l_{r}^{2}}{4 \lambda}-i l_{r} \cdot \Theta} .
$$

We can now follow [6] and do the integral over $\lambda$ and obtain a sum over Bessel functions.

$$
\begin{aligned}
\mathscr{K}_{v}(Y, Z) & =\int_{0}^{\infty} d \lambda \lambda^{v-1} e^{-Z \lambda-Y / \lambda}=2\left(\frac{Y}{Z}\right)^{\frac{v}{2}} K_{v}(2 \sqrt{Y Z}), \\
\lfloor 1\rfloor^{V}= & \frac{1}{(4 \pi)^{d / 2} \Gamma(n)} \sum_{l_{r}} e^{-i l_{r} \cdot \Theta} \mathscr{K}_{n-\frac{d}{2}-1}\left(m^{2}, \frac{l_{r}^{2}}{4}\right) .
\end{aligned}
$$

This is valid also for noninteger dimensions $d=4-2 \varepsilon$ and can, if needed, be expanded in $\varepsilon$. The triple sum can be simplified: with $\sum_{l_{r}}^{\prime} f\left(l_{r}^{2}\right)=\sum_{k>0} x(k) f(k), k=l_{r}^{2}$ and $x(k)$ is the number of times that $l_{r}^{2}=k L^{2}$. The exponential decay for large $L$ follows directly from $K_{i}(m L \sqrt{k}) \approx$ $\sqrt{\frac{\pi}{2 m L \sqrt{k}}} e^{-m L \sqrt{k}}$.

Alternatively, we can do the sum and obtain an integral over Jacobi theta functions [9]. The third Jacobi theta function is $\theta_{3}(u \mid \tau)=\sum_{n} e^{i \pi \tau n^{2}+2 \pi i u n}$. It satisfies $\theta_{3}(u+n \mid \tau)=\theta_{3}(u \mid \tau)$ and $\theta_{3}(u \mid \tau)=\frac{1}{\sqrt{-i \tau}} e^{-\pi i \frac{u^{2}}{\tau}} \theta_{3}\left(\frac{u}{\tau} \mid \frac{-1}{\tau}\right)$. Especially the latter is useful for small $\lambda$ for the tadpole integral after doing the sum

$$
\lfloor 1\rfloor^{V}=\frac{1}{(4 \pi)^{d / 2} \Gamma(n)} \int_{0}^{\infty} d \lambda \lambda^{n-\frac{d}{2}-1} e^{-\lambda m^{2}}\left[\Pi_{j=x, y, z} \theta_{3}\left(-\theta_{j} /(2 \pi) \mid i L^{2} /(4 \pi \lambda)\right)-1\right]
$$

If no twist angles are present, it becomes a cubed theta function. In the presence of twist angles, the trick with $x(k)$ of reducing the triple sum to a single sum does not work, so (3.6) is usually the better choice in that case.

As an example for the numerical size of the correction I show in Fig. 1 the relative correction to the infinite volume integral $A=\int_{V} \frac{d^{d} p}{(2 \pi)^{d}} \frac{1}{p^{2}+m^{2}}$ for two different masses as well as the result for some twist angles. 

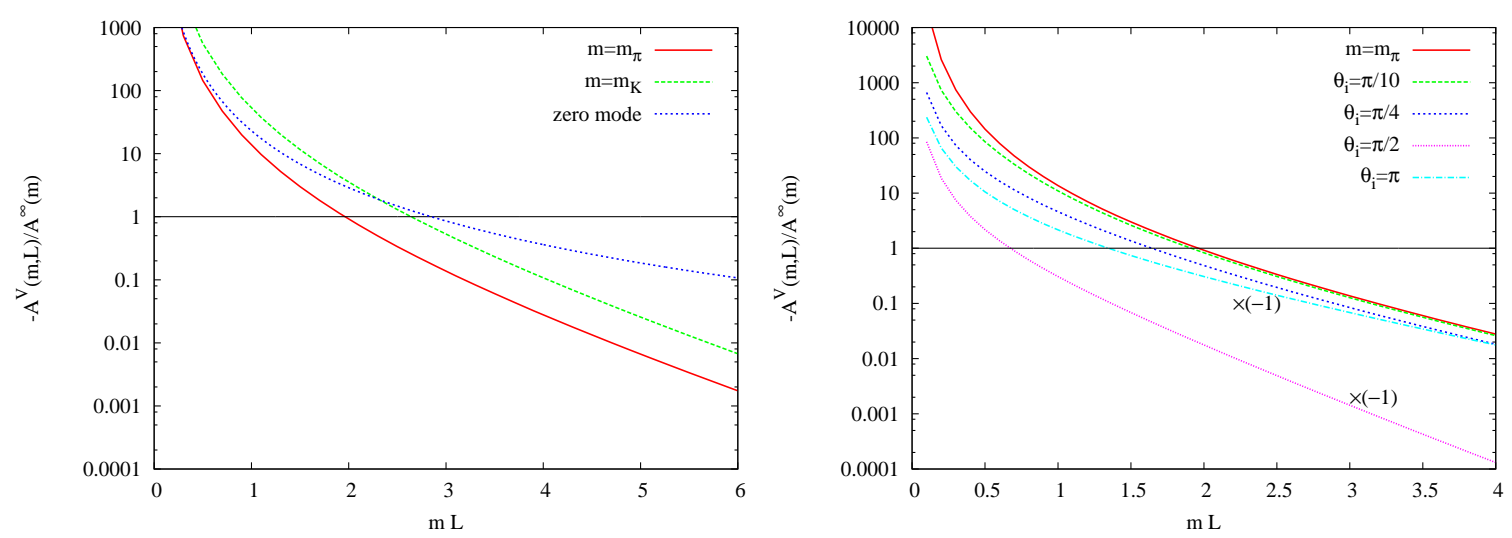

Figure 1: Left: The relative correction to the infinite volume integral $A=\int_{V} \frac{d^{d} p}{(2 \pi)^{d}} \frac{1}{p^{2}+m^{2}}$ for $\mu=0.77 \mathrm{GeV}$ and $m=m_{\pi}, m_{K}$. Also show is the contribution from the first spatial zero mode. Right: The same but with a number of twist angles $\theta_{x}=\theta_{y}=\theta_{z}=\theta_{i}$. For $\theta=\pi / 2$ all terms with an $\left(l_{r}\right)_{i}$ odd cancel, and the corrections are much smaller.

\section{More general one-loop integrals}

The methods above can be generalize to more complicated one-loop integrals as long as one stays below thresholds. In particular, for integrals with numerators $r_{\mu} r_{v}$ the steps are

- Shift with $r=\bar{r}+i l_{r} /(2 \lambda)$

- Integrals done with $\bar{r}_{\mu} \bar{r}_{v} \rightarrow \bar{r}^{2} \delta_{\mu v} / d$

- Similar for more complicated numerators

- But extra terms show up: box and twisting break Lorentz invariance

For integrals with more denominators and external momentum:

- Combine the denominators with Feynman parameters

- Shift with $r=\bar{r}+i l_{r} /(2 \lambda)+(1-x) p$

- This gives extra factors like $e^{-i(1-x) l_{r} \cdot p}$

- Center of mass system $p=(p, 0,0,0) \Longrightarrow l_{r} \cdot p=0$

- Moving frame: deal with as for twist angle

Because of the broken Lorentz invariance, there are more terms and this will lead to extra formfactors at finite volume.

\section{Sunset integrals}

I will stick to the simplest sunset integral here. The more complicated cases are treated in [7]. The simplest sunset integral is

$$
\langle\langle X\rangle\rangle=\int_{V} \frac{d^{d} r}{(2 \pi)^{d}} \frac{d^{d} s}{(2 \pi)^{d}} \frac{X}{\left(r^{2}+m_{1}^{2}\right)^{n_{1}}\left(s^{2}+m_{2}^{2}\right)^{n_{2}}\left((r+s-p)^{2}+m_{3}^{2}\right)^{n_{3}}}, .
$$


We now need to do the Poisson summation trick twice:

$$
\langle\langle X\rangle\rangle=\sum_{l_{r}, l_{s}} \int \frac{d^{d} r}{(2 \pi)^{d}} \frac{d^{d} s}{(2 \pi)^{d}} \frac{X e^{i l_{r} \cdot r} e^{i l_{s} \cdot s}}{\left(r^{2}+m_{1}^{2}\right)^{n_{1}}\left(s^{2}+m_{2}^{2}\right)^{n_{2}}\left((r+s-p)^{2}+m_{3}^{2}\right)^{n_{3}}}
$$

We stick here to the simplest case $X=1, n_{1}=n_{2}=n_{3}=1$ and $p=(p, 0,0,0) . l_{r}$ and $l_{s}$ are of the form $l_{i}=\left(0, n_{1} L, n_{2} L, n_{3} L\right)$. For the tadpole case we split off $l_{r}=0$ but here it is a little more complicated. First remove the infinite volume part with

$$
\langle\langle X\rangle\rangle=\langle\langle X\rangle\rangle^{\infty}+\langle\langle X\rangle\rangle^{V},
$$

But we also have the parts where only one of the loop momenta feels the boundary or is quantized.

$$
\langle\langle 1\rangle\rangle^{V}=\langle\langle 1\rangle\rangle_{r}+\langle\langle 1\rangle\rangle_{s}+\langle\langle 1\rangle\rangle_{t}+\langle\langle 1\rangle\rangle_{r s},
$$

The parts are defined as

$$
\langle\langle 1\rangle\rangle_{\{r, s, t, r s\}}=\left\{\sum_{l_{r}}^{\prime}, \sum_{l_{s}}^{\prime}, \sum_{l_{t}}^{\prime}, \sum_{l_{r}, l_{s}}^{\prime \prime}\right\} \times \int \frac{d^{d} r}{(2 \pi)^{d}} \frac{d^{d} s}{(2 \pi)^{d}} \frac{\left\{e^{i l_{r} \cdot r}, e^{i l_{s} \cdot s}, e^{i l_{t} \cdot(r+s)}, e^{i l_{r} \cdot r} e^{i l_{s} \cdot s}\right\}}{\left(r^{2}+m_{1}^{2}\right)\left(s^{2}+m_{2}^{2}\right)\left((r+s-p)^{2}+m_{3}^{2}\right)} .
$$

The sums are over $\left\{l_{r} \neq 0, l_{s}=0 ; l_{r}=0, l_{s} \neq 0 ; l_{t} \equiv l_{r}=l_{s} \neq 0 ; l_{r} \neq 0, l_{s} \neq 0, l_{r} \neq l_{s}\right\}$. The first three terms are those where only one-loop momentum $r, s$ or $r+s$ is quantized. The last one with both $r, s$ quantized.

\subsection{One momentum quantized}

We have sums over full momentum integrals, so we can use momentum redefinitions to relate the three first terms:

- $\left(r \leftrightarrow s, l_{r} \leftrightarrow l_{s}\right) \Longrightarrow\langle\langle 1\rangle\rangle_{s}\left(m_{1}, m_{2}, m_{3}\right)=\langle\langle 1\rangle\rangle_{r}\left(m_{2}, m_{1}, m_{3}\right)$

- $\left(r \leftrightarrow t=r-s-p, l_{t} \leftrightarrow-l_{r}, e^{i l_{t} \cdot p}=1\right) \Longrightarrow\langle\langle 1\rangle\rangle_{t}\left(m_{1}, m_{2}, m_{3}\right)=\langle\langle 1\rangle\rangle_{r}\left(m_{3}, m_{2}, m_{1}\right)$

So we only need $\langle\langle 1\rangle\rangle_{r}$. For $\langle\langle 1\rangle\rangle_{r}$ the $s$ integral is standard infinite volume:

$$
\langle\langle 1\rangle\rangle_{r}=\sum_{l_{r}}^{\prime} \int \frac{d^{d} r}{(2 \pi)^{d}} \frac{e^{i l_{r} \cdot r}}{\left(r^{2}+m_{1}^{2}\right)} \int_{0}^{1} d x \frac{\Gamma\left(2-\frac{d}{2}\right)}{(4 \pi)^{\frac{d}{2}}}\left(\bar{m}^{2}\right)^{\frac{d}{2}-2}
$$

where we can use the usual expansions $\frac{\Gamma\left(2-\frac{d}{2}\right)}{(4 \pi)^{\frac{d}{2}}}\left(\bar{m}^{2}\right)^{\frac{d}{2}-2}=\frac{1}{16 \pi^{2}}\left[\lambda_{0}-1-\log \left(\bar{m}^{2}\right)\right]+\mathscr{O}(\varepsilon)$ with $\lambda_{0}=1 / \varepsilon+\log (4 \pi)+1-\gamma$ and $\bar{m}^{2}=(1-x) m_{2}^{2}+x m_{3}^{2}+x(1-x)(r-p)^{2}$.

The part containing $\lambda_{0},\langle\langle 1\rangle\rangle_{r, A}$, should cancel in the final result (of a physical quantity), so we ignore it. We do partial integration in $x$ for the $\log \left(\bar{m}^{2}\right)$ term obtaining

$$
\langle\langle 1\rangle\rangle_{r}=\langle\langle 1\rangle\rangle_{r, A}+\langle\langle 1\rangle\rangle_{r, G}+\langle\langle 1\rangle\rangle_{r, H}, \quad\langle\langle 1\rangle\rangle_{r, G}=-\frac{1+\log \left(m_{3}^{2}\right)}{16 \pi^{2}} \bar{A}^{V}\left(m_{1}^{2}\right) .
$$

The remaining part is

$$
\langle\langle 1\rangle\rangle_{r, H}=\frac{1}{16 \pi^{2}} \sum_{l_{r}}^{\prime} \int \frac{d^{4} r}{(2 \pi)^{4}} \frac{e^{i l_{r} \cdot r}}{\left(r^{2}+m_{1}^{2}\right)} \int_{0}^{1} d x x \frac{m_{3}^{2}-m_{2}^{2}+(1-2 x)(r-p)^{2}}{\bar{m}^{2}}
$$


Bring up the denominators with ' $\alpha$ ' parameters, shift $r$, do $\tilde{r}$ integral and finally symmetrize expression, and we get (details in [7])

$$
\langle\langle 1\rangle\rangle_{r, H}=\frac{1}{\left(16 \pi^{2}\right)^{2}} \sum_{l_{r}} \int_{0}^{\infty} d \lambda_{1} d \lambda_{2} d \lambda_{3} \frac{\lambda_{3}}{\tilde{\lambda}^{2}} e^{-M^{2}}\left(m_{3}^{2}-m_{2}^{2}+\frac{\lambda_{2}-\lambda_{3}}{\tilde{\lambda}}\left(2+\frac{\lambda_{3}+\lambda_{2}}{\tilde{\lambda}} \tilde{p}^{2}\right)\right)
$$

with $M^{2}=\lambda_{1} m_{1}^{2}+\lambda_{2} m_{2}^{2}+\lambda_{3} m_{3}^{2}+\frac{\lambda_{1} \lambda_{2} \lambda_{3}}{\lambda} p^{2}+\frac{\lambda_{2}+\lambda_{3}}{\grave{\lambda}} \frac{l_{r}^{2}}{4}, \tilde{p}=\frac{i l_{r}}{2}-\lambda_{1} p$, and $\tilde{\lambda}=\lambda_{1} \lambda_{2}+\lambda_{2} \lambda_{3}+\lambda_{3} \lambda_{1}$. This contains a triple integral and a triple sum.

\subsection{Two momenta quantized: $\langle\langle 1\rangle\rangle_{r s}$}

The same general method works: Bring up denominators; Shift integration momenta; do the momentum integrals and since it is finite, set $d=4$. This leads to the results

$$
\langle\langle 1\rangle\rangle_{r s}=\frac{1}{\left(16 \pi^{2}\right)^{2}} \sum_{l_{r}, l_{s}}^{\prime \prime} \int_{0}^{\infty} d \lambda_{1} d \lambda_{2} d \lambda_{3} \tilde{\lambda}^{-2} e^{-\tilde{M}^{2}}
$$

with $\tilde{M}^{2}=\lambda_{1} m_{1}^{2}+\lambda_{2} m_{2}^{2}+\lambda_{3} m_{3}^{2}+\frac{\lambda_{1} \lambda_{2} \lambda_{3}}{\tilde{\lambda}} p^{2}+\frac{\lambda_{2}}{\bar{\lambda}} \frac{l_{r}^{2}}{4}+\frac{\lambda_{1}}{\grave{\lambda}} \frac{l_{s}^{2}}{4}+\frac{\lambda_{3}}{\bar{\lambda}} \frac{\left(l_{r}-l_{s}\right)^{2}}{4}$ and $\tilde{\lambda}=\lambda_{1} \lambda_{2}+\lambda_{2} \lambda_{3}+\lambda_{3} \lambda_{1}$. This contains a triple integral and a sextuple sum, very similar to the previous subsection.

\subsection{Preliminary numerical results}

We basically proceed as in the tadpole case but with a few extras. First we set $\lambda_{1}=\lambda x$, $\lambda_{2}=\lambda y, \lambda_{3}=\lambda(1-x-y)$. We can do the $\lambda$ integral and obtain a sextuple sum over Bessel functions. This can be reduced to a triple sum if there is no twist and we use that $p \cdot l_{r}=p \cdot l_{s}=0$, since $p$ has no components in the finite size directions. In that case we have a triple sum over $k_{1}=l_{r}^{2}$, $k_{2}=l_{s}^{2}, k_{3}=\left(l_{r}-l_{s}\right)^{2}$ and a quantity $x\left(k_{1}, k_{2} . k_{3}\right)$ that takes into account how often each set of $k_{i}$ shows up in the sextuple sum. Alternatively, the sum can be performed in terms of the Riemann or Siegel theta function:

$$
\theta^{(g)}(z \mid \tau)=\sum_{n \in \mathbb{Z}^{g}} e^{2 \pi i\left(\frac{1}{2} n^{T} \tau n+n^{T} z\right)}
$$

This function has the useful properties $\theta^{(g)}(z \mid \tau)=\theta^{(g)}\left(a z \mid a \tau a^{T}\right)$ (with $a$ and $a^{-1}$ integer) and $\theta^{(g)}\left(\tau^{-1} z \mid-\tau^{-1}\right)=\sqrt{\operatorname{det}(-i \tau)} e^{\pi i z^{T} \tau^{-1} z} \theta^{(g)}(z \mid \tau)$. The latter allows to speed up computation.

Some comments about the numerical work: Getting 5-6 digits of precision for $\langle\langle 1\rangle\rangle_{r s}$ goes fine, it takes a while but is not too bad. This method works below threshold. For the speed; typically for large $m L$ the Bessel version is fastest, while for small or medium $m L$ the theta function works fastest. The two methods always agree. Reaching a specified accuracy is easier with the theta function version. Some representative preliminary numerical results are shown in Fig. 2.

\section{Conclusions and future}

An important hurdle in two-loop Chiral Perturbation Theory at finite volume has been taken. Somewhat surprisingly, the various pieces in the sunset are all needed dependent on the inputs used. A short side-note is that the Riemann theta function in all its varieties not present in mathematica (we need its derivatives) but needs to be programmed. 

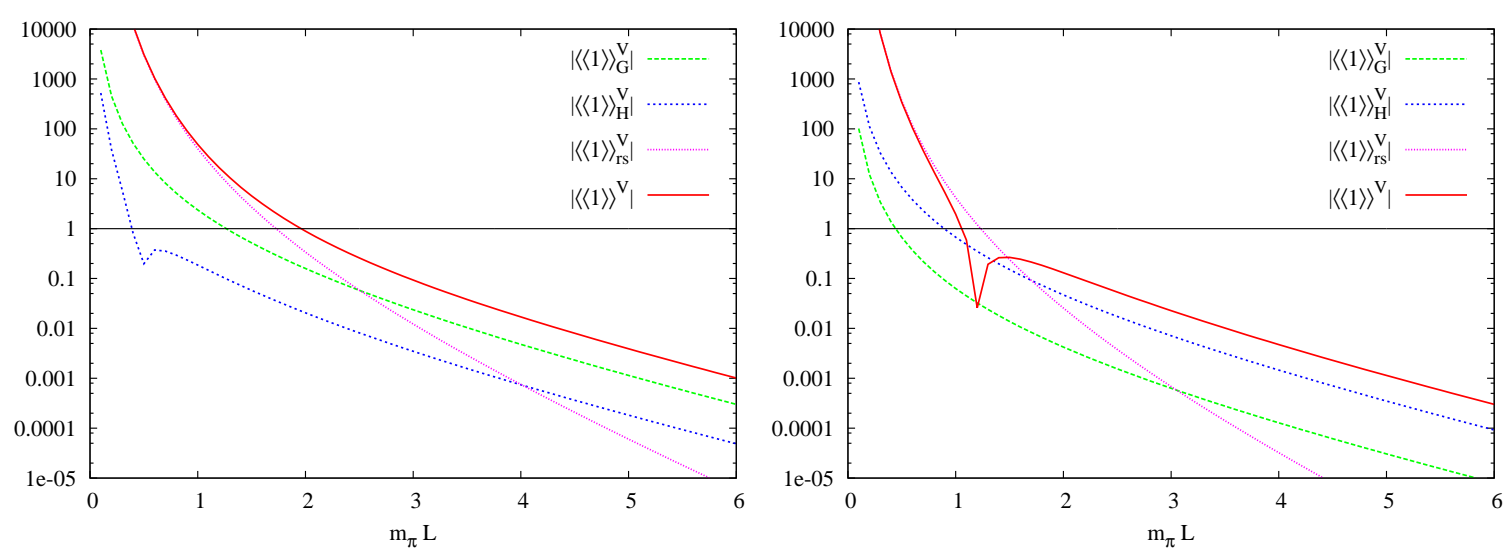

Figure 2: The simplest sunset integral and its various parts for Left: $m_{1}=m_{2}=m_{3}=m_{\pi}, p^{2}=-m_{\pi}^{2}$ (pion on shell) relative to $\langle\langle 1\rangle\rangle^{\infty}=3.738410^{-5} \mathrm{GeV}^{2}$. Right: $m_{1}=m_{2}=m_{\pi}, m_{3}=m_{K}, p^{2}=-m_{K}^{2}$ (kaon on shell) relative to $\langle\langle 1\rangle\rangle^{\infty}=6.740710^{-5} \mathrm{GeV}^{2}$. The funny bumps are not physical, we show absolute value in a log plot and the result went through zero.

The cases with numerators are in progress [7]. Moving frame and/or twisting we have not studied yet, but I see no obvious new problems appearing. The two-loop 3-flavour ChPT will need to be redone from scratch since not all integral relations at infinite volume remain valid and they were heavily used in the earlier work. This calculation is in progress.

\section{Acknowledgments}

This work is supported in part by the European Community SP4-Capacities "Study of Strongly Interacting Matter" (HadronPhysics3, Grant Agreement n. 283286), the Swedish Research Council grants 621-2011-5080 and 621-2010-3326. I also thank my collaborators Emil Boström and Timo Lähde for a pleasant collaboration.

\section{References}

[1] J. Bijnens, N. Danielsson and T. A. Lähde, Phys. Rev. D 70, 111503 (2004) [arXiv:hep-lat/0406017].

[2] J. Bijnens and T. A. Lähde, Phys. Rev. D 72, 074502 (2005) [arXiv:hep-lat/0506004].

[3] J. Bijnens, N. Danielsson and T. A. Lähde, Phys. Rev. D 73, 074509 (2006) [arXiv:hep-lat/0602003].

[4] J. Bijnens and K. Ghorbani, Phys. Lett. B 636 (2006) 51 [arXiv:hep-lat/0602019].

[5] G. Colangelo and C. Haefeli, Nucl. Phys. B 744, 14 (2006) [arXiv:hep-lat/0602017].

[6] J. Gasser and H. Leutwyler, Nucl. Phys. B 307 (1988) 763.

[7] J. Bijnens, E. Boström and T. Lähde, to be published.

[8] E. Boström, Master thesis, LU TP 13-22, Lund University

[9] D. Becirevic and G. Villadoro, Phys. Rev. D 69 (2004) 054010 [hep-lat/0311028]. 